УДК 541.138

\title{
ВОДОРОДОПРОНИЦАЕМОСТЬ ПЛЕНОЧНЫХ СПЛАВОВ Рd53Сu РАЗЛИЧНОГО ФАЗОВОГО СОСТАВА
}

\author{
() 2018 А. И. Федосеева, Н. Б. Морозова, А. В. Введенский, А. И. Донцов \\ Воронежский государственный университет, Университетская пл., 1, 394018 Воронеж, Россия \\ e-mail:mnb@chem.vsu.ru
}

Поступила в редакцию 23.03.2018

\begin{abstract}
Аннотация. Методом двухступенчатой катодно-анодной хроноамперометрии изучено влияние фазового состава пленочных сплавов $\mathrm{Pd} 53 \mathrm{Cu}$ на процессы инжекции и экстракции атомарного водорода. Исследования проведены в водном растворе $0.1 \mathrm{M} \mathrm{H}_{2} \mathrm{SO}_{4}$. Установлено, что инжекция атомарного водорода в сплав в начальный период протекает в диффузионно-фазограничном режиме, а затем - диффузионном. Найдены кинетические и диффузионные параметры фазограничного проникновения водорода и его последующей твердофазной диффузии. Выявлена роль фазового состава и кристаллографической ориентации сплава на водородопроницаемость. Показано, что перенос атомарного водорода в сплаве главным образом происходит по телу зерна, а не по межфазным границам.
\end{abstract}

Ключевые слова: катодно-анодная хроноамперометрия, $\mathrm{Pd} 53 \mathrm{Cu}, \beta$-фаза твердого раствора $\mathrm{Cu}-\mathrm{Pd}$, пленочные электроды, водородопроницаемость, фазовый состав, кристаллографическая ориентация поверхности.

DOI: https://doi.org/10.17308/kcmf.2018.20/519

\section{ВВЕДЕНИЕ}

Медно-палладиевые сплавы получили широкое применение во многих отраслях промышленности, в том числе в области очистки водорода, благодаря их высокой водородопроницаемости, стойкости к охрупчиванию и отравлению серой [1]. Преимуществом данных сплавов также является их более низкая стоимость по сравнению с чистым палладием.

Чистый водород, используемый в топливных элементах, является основным энергоносителем в водородной энергетике. Помимо того он используется в восстановительной металлургии, микрои наноэлектронике, производстве чистых материалов, химической промышленности, а также в качестве топлива для экологического транспорта и автономных энергетических установок [2-4].

Из-за необходимости выделения водорода из газовых смесей и его глубокой очистки, все больше внимания привлекают мембраны на основе палладия и его сплавов $[2,5,6]$. Наиболее высокой водородопроницаемостью обладает сплав $\mathrm{Cu}-\mathrm{Pd}$ с $X_{\mathrm{Pd}}=$ 30-55 ат.\% [7, 8], который может кристаллизоваться как в ГЦК $(\alpha$-фаза), так и в ОЦК ( $\beta$-фаза) кристал- лическую решетку [9]. Недостатки медно-палладиевых сплавов связаны с наличием эффекта дегазации, и сопряженными с ним самопроизвольными многостадийными $\beta \rightarrow \alpha$ фазовыми превращениями. Последние обусловлены дефектностью структуры и самоорганизацией возникших дефектных образований, в результате взаимодействия их между собой и водородом [10], нагревом или охлаждением сплава, содержанием в сплаве меди [11], а также степенью его деформации [12].

Сплавы системы Pd-Cu в зависимости от способа получения могут различаться по механическим характеристикам, фазовому составу, иметь разную кристаллографическую ориентацию и, как следствие, разную водородопроницаемость [13, 14]. К примеру, в [12] показано, что после деформации и последующего отжига сплав с содержанием меди 53 ат.\% имеет более низкое удельное электросопротивление и устойчив к процессам $\beta \rightarrow \alpha$ фазового перехода в бульшем интервале температур. Однако при длительном отжиге деформированный сплав с содержанием меди $\leq 10$ ат.\% характеризуется бульшим значением удельного электросопротивления и повышенной микротвердостью образ- 
ца [15]. Сплавы, полученные магнетронным распылением [16], обладают высокой прочностью и при этом достаточно пластичны.

В данной работе в качестве электродов использовали тонкие, несколько мкм, металлические фольги, полученные методом магнетронного распыления в вакууме сплава $\mathrm{Cu}-\mathrm{Pd}$.

Цель работы: выявление роли фазового состава и кристаллографической ориентации твердого раствора $\mathrm{Cu}-\mathrm{Pd}$ в формировании параметров электрохимических процессов экстракции и инжекции атомарного водорода в сплав из сернокислого водного раствора.

\section{ЭКСПЕРИМЕНТАЛЬНАЯ ЧАСТЬ}

Образцы, растворы, аппаратура. В качестве электродов использовали медно-палладиевые пленочные электроды состава 53 ат.\% $\mathrm{Cu}$ и 47 ат.\% $\mathrm{Pd}$, представляющие собой твердый раствор замещения. Образцы получали методом магнетронного распыления в вакууме, где исходный вакуум в рабочей камере $10^{-3}$ Па, а давление рабочего газа (Ar) составило $1.6 \cdot 10^{-1}$ Па. Конденсацию образцов проводили при постоянном токе 0.5 А и ускоряющем напряжении 500 В на подложки из оксидированных монокристаллических пластин кремния $\left(\mathrm{SiO}_{2} / \mathrm{Si}\right)$ при температуре $T_{\text {sub }}=700 \mathrm{~K}$. Скорость конденсации составляла $1 \mathrm{Hм} / \mathrm{c}$ [16]. Образцы 1 и 2 представляют собой $100 \%$-ную $\beta$-фазу твердого раствора $\mathrm{Cu}-\mathrm{Pd}$, но разной кристаллографической ориентации. Для выявления влияния содержания $\beta$-фазы привлечены данные, полученные в [17] для электрода, представляющего смесь $\alpha$ - и $\beta$-фаз (образец 3). Состав фаз в образце 3 на разных сторонах поверхности образцов несколько отличался: внешняя сторона поверхности содержит 52, а внутренняя - 41 ат.\% $\beta$-фазы. Толщины $L$ исследуемых электродов, а именно 1,2 и 3 , составляли 11 , 8 и 2.6 мкм соответственно.

Пленочные электроды имеют две стороны поверхности - внутреннюю, обращенную к подложке, и внешнюю. Поскольку эти поверхности отличаются шероховатостью, субструктурой и фазовым составом [18], электрохимические исследования проводились на обеих сторонах. Для проведения электрохимических измерений использовали электрод из спектрально чистого графита, на который с помощью токопроводящего графитового клея крепили образец металлической пленки. Токопроводящий клей представляет собой раствор измельченного спектрально чистого графита в толуоле. В качестве связывающего компонента использовался пенополистирол. Со- противление полученного клея не превышало 0.05 Ом'м. В отдельных опытах установлено, что графитовый клей не оказывает влияния на процессы выделения и ионизации атомарного водорода. Площадь пленочных образцов не превышала $1 \mathrm{~cm}^{2}$; каждый образец использовался только один раз.

Исследования проводили методами циклической вольтамперометрии и двухступенчатой катодно-анодной хроноамперометрии (ХАГ) в растворе $0.1 \mathrm{M} \mathrm{H}_{2} \mathrm{SO}_{4}$ (ос.ч), деарированном химически чистым аргоном. Подробное описание используемых методов представлено в $[17,19]$. Электрохимические измерения проводили с использованием потенциостата IPC-Compact; потенциалы пересчитаны относительно ст.в.э. Потенциал наводороживания $\left(E_{\mathrm{c}}\right)$ составлял $-0.08 \mathrm{~B}$. Потенциал ионизации $E_{\mathrm{a}}$ соответствовал потенциалу пика ионизации атомарного водорода, который находили по предварительно полученным вольтамперограммам. Значения $E_{\text {a }}$ для разных образцов несколько отличались. Время наводороживания $t_{\mathrm{c}}$ не превышало $10 \mathrm{c}$, что исключало возможность образования гидрида палладия, поскольку отношение $\mathrm{H} / \mathrm{Pd}$ в исследуемых образцах не превышало 0.022 .

\section{РЕЗУЛЬТАТЫ И ИХ ОБСУЖДЕНИЕ}

Рентгеновские дифрактограммы для пленочных образцов $\mathrm{Pd}-\mathrm{Cu}$, представленны на рисунке 1 [16].

Образцы 1 и 2 содержат только $\beta$-фазу твердого раствора и несколько отличаются между собой кристаллографической ориентацией. Образец 1 имеет более плотноупакованную структуру, что характеризуется наличием двух четких пиков $<111 \beta>$ и $<211 \beta>$. Для образца 2 также наблюдаются 2 пика, соответствующие $\beta$-фазе твердого раствора, один из которых, как и для образца $1-<211 \beta>$, а второй, с более высокой амплитудой $-<210 \beta>$. Отсюда можно заключить, что образец 1 структурно более упорядочен, чем 2. Для образца 3 характерно содержание как $\alpha$-, так и $\beta$-фазы твердого раствора. Здесь выявляется пик $<111 \alpha>$, т. е. преобладает другой тип решетки - ГЦК с ориентацией $<111>$.

Многоцикловая катодно-анодная хроноамперометрия. Типичные хроноамперограммы (ХАГ), полученные на исследуемых пленочных электродах представлены на рис. 2.

С увеличением времени наводороживания $t$ с от 1 до 10 с происходит постепенный рост скорости ионизации атомарного водорода. Характер спада тока на внешней и внутренней поверхностях (в пределах одной пленки) не изменяется, вид 


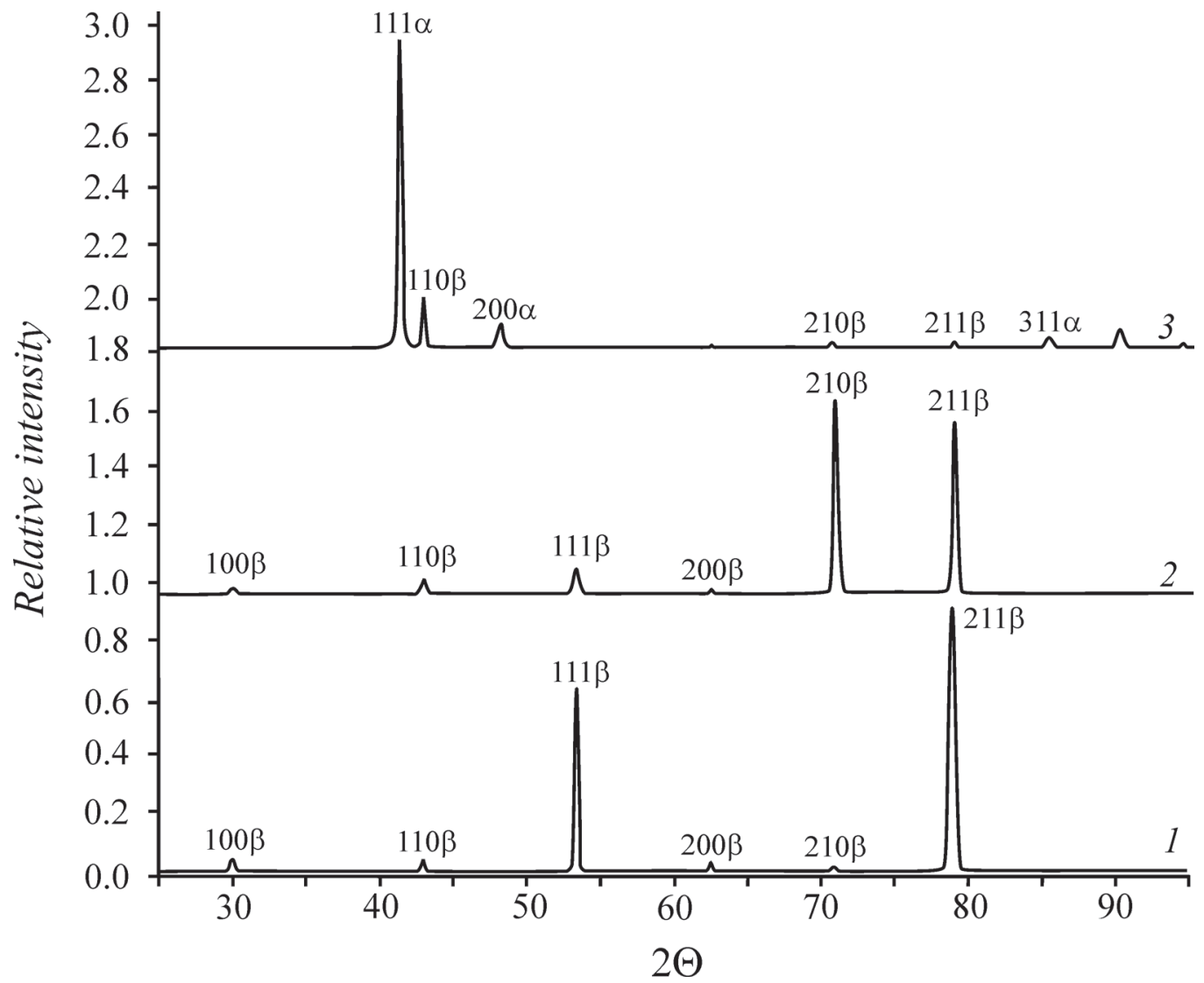

Рис. 1. Рентгеновские дифрактограммы исследуемых образцов фольг 1, 2 и 3 сплава $\mathrm{Pd} 53 \mathrm{Cu}$ [Fig. 1. X-ray diffraction patterns of the investigated samples of foils 1, 2 and 3 of the Pd53Cu alloy]
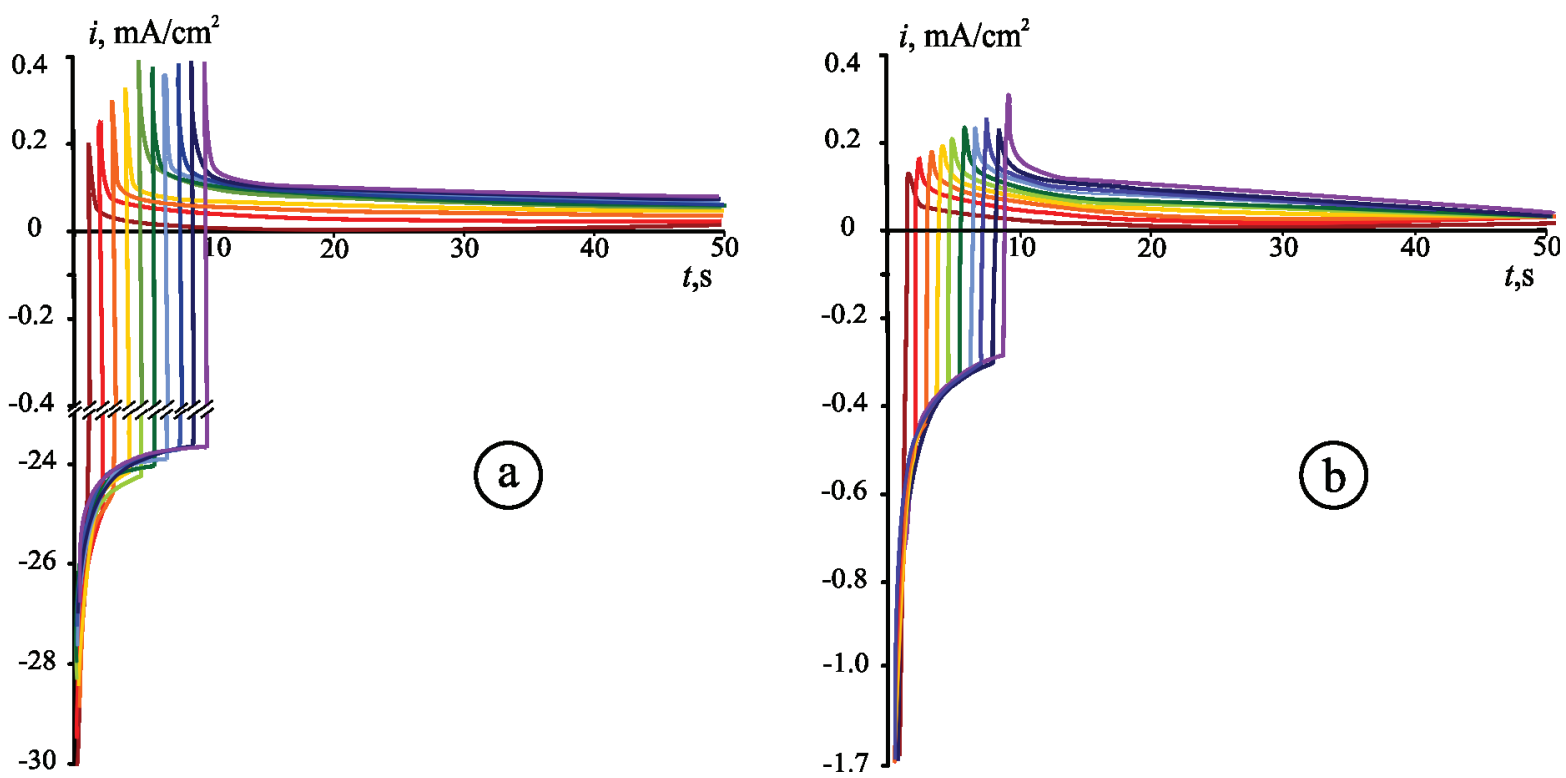

Рис. 2. Двухступенчатые катодно-анодные хроноамперограммы пленочных образцов состава Pd53Cu: a) образец $1 ; b)$ образец 2

[Fig. 2. Two-stage cathodic-anodic chronoamperograms of Pd53Cu film: $a$ ) sample $1 ; b$ ) sample 2] 
катодных и анодных ХАГ аналогичен. Величины катодных токов для образца 1 в 15-20 раз превышают аналогичные значения для образца 2. Различия в анодных спадах тока значительно меньше, а максимальные значения $i_{\mathrm{a}}$ отличаются менее чем в 2 раза. Необходимо отметить, что на обоих образцах основной анодный спад тока происходит в течение первых $10 \mathrm{c}$, а затем снижение $i$ происходит более плавно. Характер катодного спада тока не зависит от величины $t_{\mathrm{c}}$, а его конечная величина достигает одного и того же значения.

Обработку полученных катодных и анодных хроноамперограмм проводили по методике, описанной в [20]. Общее уравнение, описывающее катодный спад тока в режиме смешанной диффузионно-фазограничной кинетики, имеет вид:

$i_{\mathrm{c}}\left(t ; \eta^{\mathrm{c}}\right)=i_{\mathrm{c}}^{\infty}\left(\eta^{\mathrm{c}}\right)+\frac{F \overleftarrow{k}\left[c_{\overline{\mathrm{H}}}^{\mathrm{s}}\left(\eta^{\mathrm{c}}\right)-c_{\overline{\mathrm{H}}}^{\mathrm{e}}\right]}{\left(1+\frac{\overleftarrow{k} L}{2 D}\right)} \cdot \mathrm{e}^{-\frac{\overleftarrow{k} t}{\left(1+\frac{\overleftarrow{k} L}{2 D}\right) L}}$

После ряда преобразований и с учетом, что $(\stackrel{\leftarrow}{k} L / 2 D)<<1$, когда значительно заторможен процесс внедрения водорода в пленку:

$$
i_{c}\left(t ; \eta^{\mathrm{c}}\right)=i_{\mathrm{c}}^{\infty}\left(\eta^{\mathrm{c}}\right)+F \overleftarrow{k}\left[c_{\overline{\mathrm{H}}}^{\mathrm{s}}\left(\eta^{\mathrm{c}}\right)-c_{\overline{\mathrm{H}}}^{\mathrm{e}}\right] \cdot \mathrm{e}^{-\frac{\overleftarrow{k} t}{L}},
$$

тогда как, при $(\overleftarrow{k} L / 2 D)>>1$ кинетика инжекции атомарного водфрода диффузионная:

$$
\begin{gathered}
\ln \left[i_{\mathrm{c}}\left(t ; \eta^{\mathrm{c}}\right)-i_{\mathrm{c}}^{\infty}\left(\eta^{\mathrm{c}}\right)\right]= \\
=\ln \left[\frac{2 F D}{L}\left[c_{\overline{\mathrm{H}}}^{\mathrm{s}}\left(\eta^{\mathrm{c}}\right)-c_{\overline{\mathrm{H}}}^{\mathrm{e}}\right]\right]-\frac{2 D t}{L^{2}} .
\end{gathered}
$$

Здесь $D$ - коэффициент диффузии атомарного водорода в твердой фазе, далее обозначаемого как $\overline{\mathrm{H}}, \stackrel{\overleftarrow{k}}{-}$ - константа скорости экстракции атомарного водорода, $\Delta c_{\overline{\mathrm{H}}}=\left[c_{\overline{\mathrm{H}}}^{\mathrm{s}}\left(\eta^{\mathrm{c}}\right)-c_{\overline{\mathrm{H}}}^{\mathrm{e}}\right]-$ изменение концентрации $\overline{\mathrm{H}}$, где $c_{\overline{\mathrm{H}}}^{\mathrm{s}}-$ молярная концентрация $\overline{\mathrm{H}}$ в приповерхностном слое пленки, а $c_{\overline{\mathrm{H}}}^{\mathrm{e}}-$ его равновесная концентрация в пленке. По величине отрезка, отсекаемого на оси ординат $(a)$, рассчитано $\Delta c_{\overline{\mathrm{H}}}=\frac{\mathrm{e}^{a}}{F \stackrel{\leftarrow}{k}}$, а из наклона линейных зависимостей (b) с привлечением (2) и (3) вычислены значения $\overleftarrow{k}=b L$ и $D=\frac{b L^{2}}{2}$.
Кинетические параметры, а именно константа скорости инжекции атомарного водорода и константа фазограничного равновесия, рассчитываются по формулам (4) и (5) соответственно:

$$
\begin{gathered}
K=\vec{k} / \stackrel{\leftarrow}{k}, \\
K_{D}\left(\eta_{\mathrm{c}}\right)=D^{1 / 2}\left[c_{\overline{\mathrm{H}}}^{\mathrm{s}}\left(\eta^{\mathrm{c}}\right)-c_{\overline{\mathrm{H}}}^{\mathrm{e}}\right]= \\
=D^{1 / 2} K\left[\Theta_{\overline{\mathrm{H}}}\left(\eta^{\mathrm{c}}\right)-\Theta_{\overline{\mathrm{H}}}^{\mathrm{e}}\right] .
\end{gathered}
$$

Здесь $\Theta_{\overline{\mathrm{H}}}\left(\eta^{c}\right)$ - степень заполнения внешней поверхности пленки атомарным водородом при катодном перенапряжении $\eta^{\mathrm{c}}, \Theta_{\overline{\mathrm{H}}}^{\mathrm{e}}$ - равновесная степень заполнения поверхности пленки.

Для обработки результатов использовали хроноамперограммы, полученные при $t_{\mathrm{c}}=10 \mathrm{c}$; пример линеаризации катодных спадов тока представлен на рис. 3.

Наличие двух линейных участков на $i, t$-кривых при малых временах $(t \leq 3 \mathrm{c})$ и при больших $(t \geq 4 \mathrm{c})$ соответствует разным кинетическим режимам инжекции водорода. Можно полагать, что при малых временах процесс протекает в режиме смешанной фазогранично-диффузионной кинетики, которая со временем переходит в диффузионную. Для образца 1 характерно более высокое значение начального катодного тока, чем для образца 2. Характер спада тока одинаков для обоих образцов.

На основе полученных данных по формулам (2), (3), (5) и (2)-(4) рассчитывались параметры водородопроницаемости, представленные соответственно в табл. 1 и 2.

Параметры диффузионного процесса, представленные в табл.1, позволяют оценить влияние фазового состава, а также стороны исследуемых образцов, на коэффициент диффузии атомарного водорода и водородопроницаемость. Влияние толщины пленочных образцов проявляется, как известно [21], лишь при $L \leq 1$ мкм, где происходит основное изменение размеров кристаллитов. Влияние стороны поверхности, прежде всего, сказывается на коэффициенте диффузии водорода в образцах с большей долей $\beta$-фазы. Сравнение пленочных образцов разного фазового состава, показывает, что значение $D$ увеличивается на образцах $100 \% \beta$-фазы в 15-20 раз, в то время как концентрация атомарного водорода снижается примерно вдвое. Как результат, между всеми образцами наблюдается различие в параметрах водородопроницаемости. Поскольку с увеличением доли $\beta$-фазы в сплаве облегчается инжекция водорода, то можно предположить, что водород главным образом перемещается по телу зерна, а не по межзеренным границам. 

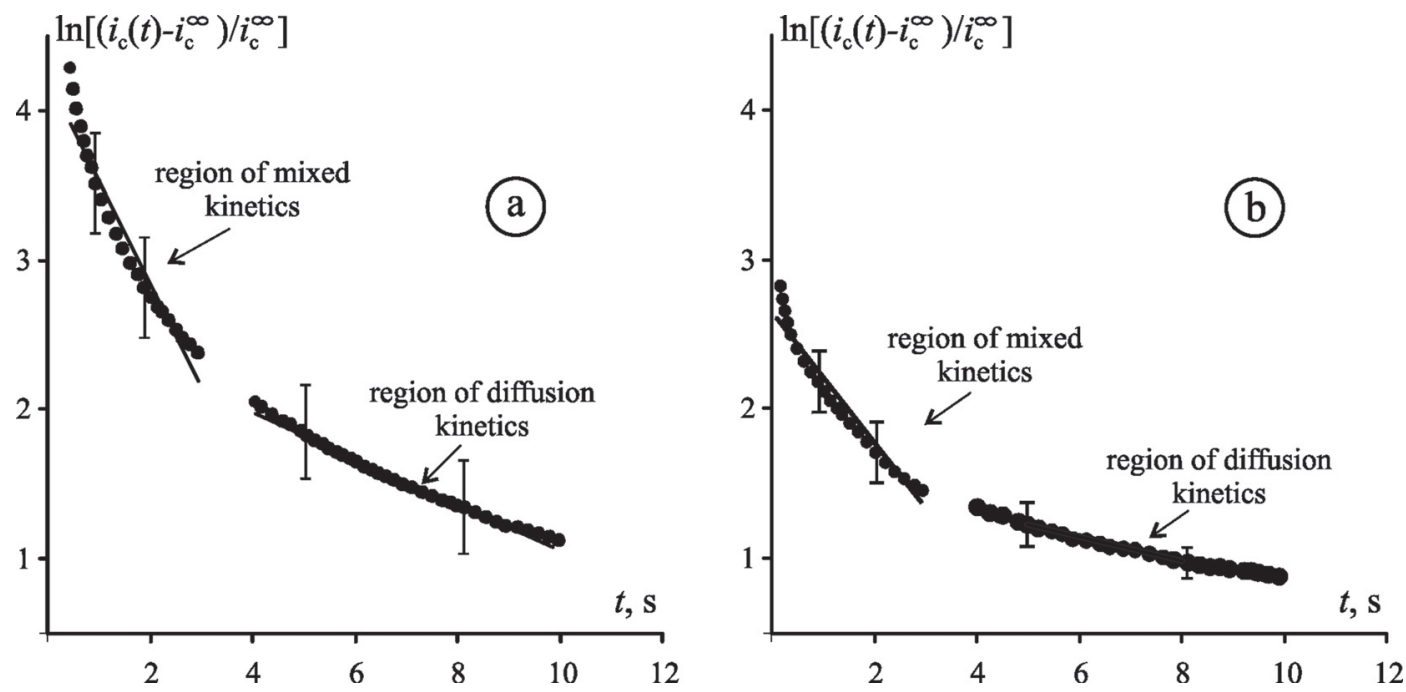

Рис. 3. Линеаризованные катодные хроноамперограммы для образеца $1(a)$ и $2(b)$

[Fig. 3. Linearized cathodic chronoamperograms for sample $1(a)$ and $2(b)$ ]

Таблица 1. Диффузионные параметры процесса катодной инжекции атомарного водорода в пленочные электроды $\mathrm{Pd} 53 \mathrm{Cu}$ (числитель - внутренняя, знаменатель - внешняя сторона пленки)

[Table 1. Diffusion parameters of the cathodic injection of atomic hydrogen in the film $\mathrm{Pd} 53 \mathrm{Cu}$ electrodes for internal (num.) and outer (denom.) side of the film]

\begin{tabular}{|c|c|c|c|c|}
\hline $\begin{array}{c}\text { Образец } \\
\text { [Sample] }\end{array}$ & $X_{\beta}, \%$ & $\begin{array}{c}D \cdot 10^{9}, \mathrm{~cm}^{2} / \mathrm{c} \\
{\left[D \cdot 10^{9}, \mathrm{~cm}^{2} / \mathrm{s}\right]}\end{array}$ & $\begin{array}{c}\Delta c_{\overline{\mathrm{H}}} \cdot 10^{5}, \text { моль } / \mathrm{cm}^{3} \\
{\left[\Delta c_{\overline{\mathrm{H}}} \cdot 10^{5}, \mathrm{~mole} / \mathrm{cm}^{3}\right]}\end{array}$ & $\begin{array}{c}K_{D} \cdot 10^{9}, \text { моль } / \mathrm{cm}^{2} \cdot \mathrm{c}^{1 / 2} \\
{\left[K_{D} \cdot 10^{9}, \mathrm{~mole} / \mathrm{cm}^{2} \cdot \mathrm{s}^{1 / 2}\right]}\end{array}$ \\
\hline 1 & $\frac{100}{100}$ & $\frac{55.10 \pm 0.11}{45.80 \pm 0.74}$ & $\frac{4.24 \pm 0.05}{4.31 \pm 0.05}$ & $\frac{9.03 \pm 0.09}{5.22 \pm 0.10}$ \\
\hline 2 & $\frac{100}{100}$ & $\frac{39.4 \pm 0.5}{36.7 \pm 0.3}$ & $\frac{4.45 \pm 0.03}{4.22 \pm 0.05}$ & $\frac{7.87 \pm 0.06}{7.59 \pm 0.08}$ \\
\hline 3 & $\frac{41}{52}$ & $\frac{2.84 \pm 0.09}{2.85 \pm 0.01}$ & $\frac{8.79 \pm 0.21}{9.83 \pm 1.36}$ & $\frac{4.68 \pm 1.34}{5.24 \pm 0.81}$ \\
\hline
\end{tabular}

Таблица 2. Кинетические параметры процесса катодной инжекции водорода в пленочные электроды $\mathrm{Pd53Cu}$ (числитель - внутренняя, знаменатель - внешняя сторона пленки)

[Table 2. Kinetic parameters of the cathodic injection of hydrogen into the film $\mathrm{Pd} 53 \mathrm{Cu}$ electrodes for internal (num.) and outer side of the film (denom.)]

\begin{tabular}{|c|c|c|c|c|}
\hline $\begin{array}{c}\text { Образец } \\
\text { [Sample] }\end{array}$ & $X_{\beta}, \%$ & $\begin{array}{c}\stackrel{\leftarrow}{k} \cdot 10^{4}, \mathrm{~cm} / \mathrm{c} \\
\left.\stackrel{\leftarrow}{k} \cdot 10^{4}, \mathrm{~cm} / \mathrm{s}\right]\end{array}$ & $\begin{array}{c}\vec{k} \cdot 10^{8}, \text { моль } / \mathrm{cm}^{2} \cdot \mathrm{c} \\
{\left[\vec{k} \cdot 10^{8}, \mathrm{~mole} / \mathrm{cm}^{2} \cdot \mathrm{s}\right]}\end{array}$ & $\begin{array}{c}K \cdot 10^{5}, \text { моль/см} \\
{\left[K \cdot 10^{5}, \mathrm{~mole} / \mathrm{cm}^{3}\right]}\end{array}$ \\
\hline 1 & $\frac{100}{100}$ & $\frac{5.38 \pm 0.02}{5.65 \pm 0.04}$ & $\frac{4.61 \pm 0.06}{3.90 \pm 0.07}$ & $\frac{7.32 \pm 0.08}{6.53 \pm 0.08}$ \\
\hline 2 & $\frac{100}{100}$ & $\frac{3.54 \pm 0.02}{4.13 \pm 0.01}$ & $\frac{1.62 \pm 0.01}{1.71 \pm 0.02}$ & $\frac{4.58 \pm 0.03}{4.10 \pm 0.04}$ \\
\hline 3 & $\frac{41}{52}$ & $\frac{2.76 \pm 1.69}{2.76 \pm 0.04}$ & $\frac{3.73 \pm 1.22}{4.17 \pm 0.64}$ & $\frac{13.31 \pm 3.62}{14.87 \pm 2.30}$ \\
\hline
\end{tabular}


Из анализа значений кинетических параметров (табл. 2) следует, что имеет место различие констант скоростей экстракции атомарного водорода для образцов разного фазового состава. Так, если для образцов одинакового фазового состава (1 и 2) эти различия незначительны, то для образца 3 значения $\overleftarrow{k}$ отличаются в несколько раз, что может указывать на наличие большего числа дефектов. В целом же кинетические параметры процесса оказались очень мало чувствительны к фазовому составу сплава. Наблюдаемое уменьшение константы скорости инжекции для образца 2 скорее всего связано с различием в кристаллографической ориентации поверхности, которая может оказать влияние именно на стадию фазограничного проникновения атомарного водорода. В то же время, диффузионные характеристики процесса практически не зависят от индекса Миллера.

Анодные хроноамперограммы характеризуют процесс экстракции атомарного водорода. Ток практически полностью спадает за 100 с, после чего достигает своего стационарного значения. Обработка анодных спадов тока проводилась с использованием выражений, представленных в [20]. Общее уравнение, описывающее полную анодную ХАГ процесса экстракции атомарного водорода, имеет вид:

$$
\begin{aligned}
& i_{\mathrm{a}}(t)=i_{\mathrm{a}}^{\infty}+\frac{2 F D}{L}\left[c_{\overline{\mathrm{H}}}^{\mathrm{s}}\left(\eta^{\mathrm{c}}\right)-c_{\overline{\mathrm{H}}}^{\mathrm{e}}\right] \times \\
& \times\left(\mathrm{e}^{-\frac{\pi^{2} D\left(t-t_{\mathrm{c}}\right)}{4 L^{2}}}-\mathrm{e}^{-\frac{\pi^{2} D\left(2 t-t_{\mathrm{c}}\right)}{4 L^{2}}}\right) .
\end{aligned}
$$

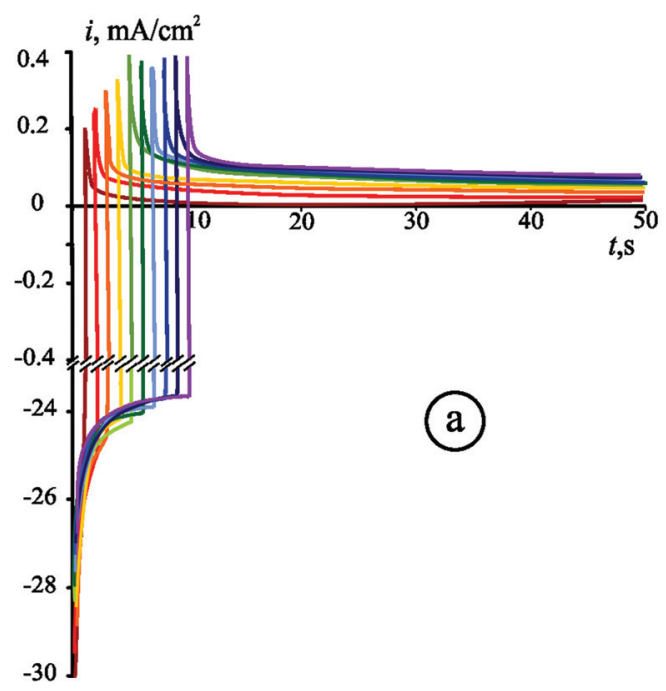

При достаточно заметных значениях $t$, сопоставимых с $t_{\mathrm{c}}$, для нахождения $D$ используется упрощенное уравнение:

$$
\ln \left(\frac{i_{\mathrm{a}}(t)-i_{\mathrm{a}}^{\infty}}{i_{\mathrm{a}}\left(t_{\mathrm{c}}\right)-i_{\mathrm{a}}^{\infty}}\right) \approx-\frac{\pi^{2} D\left(t-t_{\mathrm{c}}\right)}{4 L^{2}} .
$$

Далее, с использованием полученного по (7) значения $D$, проводится графическая линеаризация полного уравнения (6), представленного в виде:

$$
\begin{gathered}
\ln \left[i_{\mathrm{a}}(t)-i_{\mathrm{a}}^{\infty}\right]=\ln \left(\frac{2 F D}{L}\left[c_{\overline{\mathrm{H}}}^{\mathrm{s}}\left(\eta^{\mathrm{c}}\right)-c_{\overline{\mathrm{H}}}^{\mathrm{e}}\right]\right)+ \\
+\ln \left(\mathrm{e}^{-\frac{\pi^{2} D\left(t-t_{\mathrm{c}}\right)}{4 L^{2}}}-\mathrm{e}^{-\frac{\pi^{2} D\left(2 t-t_{\mathrm{c}}\right)}{4 L^{2}}}\right) .
\end{gathered}
$$

Пример линеаризации анодных спадов тока с привлечением уравнений (7) и (8) представлен на рис. 4.

Используемый метод теоретического моделирования не позволяет, к сожалению, опытным путем разделить параметры фазограничной и диффузионной стадии. Поэтому соответствующие характеристики рассчитаны в рамках модели смешанной диффузионно-фазограничной кинетики. Значения найденных по уравнениям (7) и (8) диффузионных параметров представлены в табл. 3 .

Значения параметров водородопроницаемости, полученные по анодным спадам тока, также как и по катодным, увеличиваются по мере роста доли $\beta$-фазы в сплаве, и мало чувствительны к размерам кристаллитов [21]. Так, коэффициент диффузии $D$ и константа водородопроницаемости $K_{D}$ увеличи-

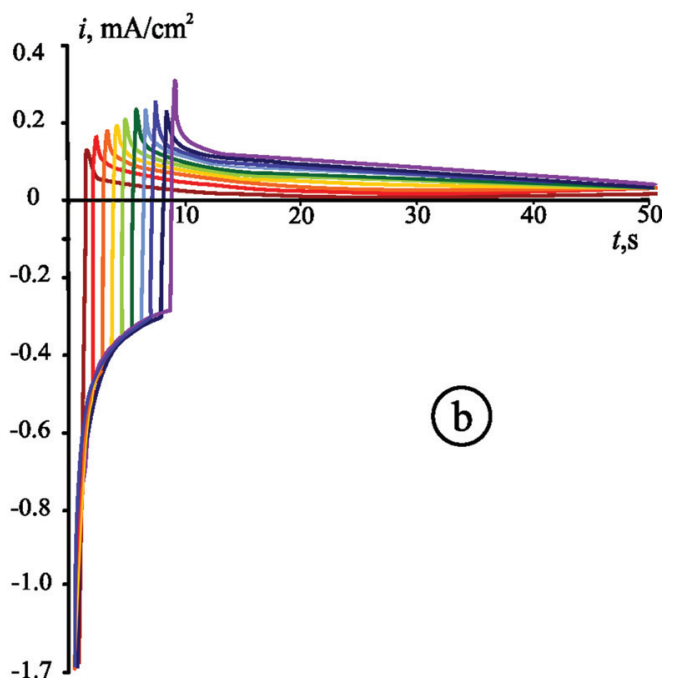

Рис. 4. Линеаризованные анодные хроноамперограммы

[Fig. 4. Linearized anodic chronoamperograms] 
Таблица 3. Характеристики диффузионного переноса атомарного водорода через пленку сплавов $\mathrm{Pd} 53 \mathrm{Cu}$, полученные в условиях анодной поляризации предварительно наводороженных образцов разного фазового состава

[Table 3. Characteristics of the diffusion transfer of atomic hydrogen through the film of Pd53Cu alloys obtained under the conditions of anodic polarization of previously hydrogenated samples with different phase composition]

\begin{tabular}{|c|c|c|c|c|c|c|c|c|}
\hline \multirow{2}{*}{$\begin{array}{l}\text { Образец } \\
\text { [Sample] }\end{array}$} & \multicolumn{2}{|c|}{$X_{\beta}, \%$} & \multicolumn{2}{|c|}{$\begin{array}{c}D \cdot 10^{9}, \mathrm{~cm}^{2} / \mathrm{c} \\
{\left[D \cdot 10^{9}, \mathrm{~cm}^{2} / \mathrm{s}\right]}\end{array}$} & \multicolumn{2}{|c|}{$\begin{array}{c}\Delta c_{\overline{\mathrm{H}}} \cdot 10^{5}, \text { моль } / \mathrm{cm}^{3} \\
{\left[\Delta c_{\overline{\mathrm{H}}} \cdot 10^{5}, \mathrm{~mole} / \mathrm{cm}^{3}\right]}\end{array}$} & \multicolumn{2}{|c|}{$\begin{array}{c}K_{D} \cdot 10^{9}, \text { моль } / \mathrm{cm}^{2} \cdot \mathrm{c}^{1 / 2} \\
{\left[K_{D} \cdot 10^{9}, \mathrm{~mole} / \mathrm{cm}^{2} \cdot \mathrm{s}^{1 / 2}\right]}\end{array}$} \\
\hline & $\begin{array}{c}\text { внутр. } \\
\text { [internal] }\end{array}$ & внеш. & $\begin{array}{c}\text { внутр. } \\
\text { [internal] }\end{array}$ & $\begin{array}{c}\text { внеш. } \\
\text { [external] }\end{array}$ & $\begin{array}{c}\text { внутр. } \\
\text { [internal] }\end{array}$ & $\begin{array}{c}\text { внеш. } \\
\text { [external] }\end{array}$ & $\begin{array}{c}\text { внутр. } \\
\text { [internal] }\end{array}$ & $\begin{array}{c}\text { внеш. } \\
\text { [external] }\end{array}$ \\
\hline 1 & 100 & 100 & $26.8 \pm 0.3$ & $14.5 \pm 0.1$ & $5.66 \pm 0.08$ & $6.67 \pm 0.08$ & $8.1 \pm 0.1$ & $8.06 \pm 0.07$ \\
\hline 2 & 100 & 100 & $26.2 \pm 0.4$ & $12.4 \pm 0.1$ & $4.05 \pm 0.04$ & $6.96 \pm 0.04$ & $5.8 \pm 0.1$ & $7.80 \pm 0.03$ \\
\hline 3 & 41 & 52 & $1.1 \pm 0.2$ & $0.9 \pm 0.1$ & $11.52 \pm 7.74$ & $10.80 \pm 2.47$ & $3.8 \pm 1.8$ & $3.22 \pm 0.37$ \\
\hline
\end{tabular}

ваются для образцов 1 и 2, по сравнению с образцом 3 , а концентрация атомарного водорода $\Delta c_{\overline{\mathrm{H}}}$ в сплавах уменьшается.

Из сравнения результатов, полученных по катодным и анодным спадам тока, следует, что значения $D$, найденные по анодным спадам, почти вдвое меньше, чем по катодным. Это может быть связано с тем, что часть атомарного водорода захватывается в ловушечных дефектах сплава, а потому не удается извлечь полностью весь внедренный водород.

\section{ВЫВОДЫ}

1. Увеличение доли $\beta$-фазы в твердом растворе пленочного электрода $\mathrm{Pd} 53 \mathrm{Cu}$ приводит, вне зависимости от толщины образца, к заметному возрастанию коэффициента диффузии и некоторому снижению концентрации атомарного водорода в сплаве, а, как следствие - увеличению его водородопроницаемости.

2. Фазовый состав сплава практически не оказывает влияния на кинетические параметры процесса, прежде всего - константы скоростей прямой и обратной стадий фазограничного обмена. Кристаллографическая ориентация граней у сплавов одного и того же фазового состава сказывается в параметрах стадии фазограничного проникновения.

3. Диффузионные параметры водородопроницаемости, рассчитанные по катодным и анодным спадам тока несколько различаются, но схожим образом меняются при увеличении доли $\beta$-фазы в пленочных электродах $\mathrm{Pd}-\mathrm{Cu}$ одного химического состава.

4. Данные, полученные на образцах сплава Pd$53 \mathrm{Cu}$ различного фазового состава, косвенно подтверждают преимущественный перенос водорода по телу зерна, а не по межфазным границам.

\section{СПИСОК ЛИТЕРАТУРЫ}

1. Morgan Allemand, Manuel. H. Martin, David Reyter, Lionel Roué, Daniel Guay, Carmen Andrei, Gianluigi A. Botton // J. Electrochimica Acta, 2011, vol. 56, p. 73977403. DOI: 10.1016/j.electacta.2011.05.052

2. Словецкий Д. И., Чистов Е. М., Рошан Н. Р. // Международный научный журнал Альтернативная энергетика и экология, 2004, № 1, с. 43-46.

3. Fuwu Yan, Lei Xu, Yu Wang // J. Renewable and Sustainable Energy Reviews, 2018, vol. 82, № 1, p. 14571488. DOI: $10.1016 /$ j.rser.2017.05.227

4. Alessandro Cappelletti, Francesco Martelli // J. Hydrogen Energy, 2017, vol. 42, p. 10513-10523.

5. Zhang Ke, Gade Sabina K., Douglas Way J. // J. embrane Science, 2012, vol. 403-404, p. 78-83. DOI: 10.1016/j.memsci.2012.02.025

6. Rahimpour M. R., Samimi F., Babapoor A., Tohidian T., Mohebi S. // J. Chemical Engineering and Processing, 2017, vol. 121, p. 24-49. DOI: 10.1016/j.cep.2017.07.021

7. Morreale B. D., Ciocco M. V., Howard B. H., Killmeyer R. P., Cugini A. V., Enick R. M. // J. Membrane Science, 2004, vol. 241, p. 219-224. DOI: 10.1016/j.memsci.2004.04.033

8. Ramesh Sharma, Yamini Sharma // J. Hydrogen Energy, 2015, vol. 40, p. 14885-14899. DOI: 10.1016/ j.ijhydene.2015.09.016

9. Морозов М. М., Потекаев А. И., Клопотов А. А., Маркова Т. Н., Клопотов В. Д. // Известия высших учебных заведений. Черная металлургия, 2015, т. 58, № 8, c. 589-596. DOI: 10.17073/0368-0797-2015-8-589-596

10. Авдюхина В. М., Ревкевич Г. П., Кацнельсон А. А. // Поверхность. Рентгеновские, синхротронные и нейтронные исследования, 2006, № 12, с. 15-22.

11. Волков А. Ю., Казанцев В. А., Коуров Н. И., Кругликов Н. А. // Физика металлов и металловедение, 2008, т. 106, № 4, с. 355-366. DOI: https://doi.org/10.1134/ S0031918X08100037

12. Волков А. Ю., Кругликов Н. А. // Физика металлов и металловедение, 2008, т. 105, № 2, с. 215-224. DOI: https://doi.org/10.1007/s11508-008-2013-5

13. Nayebossadri S., Speight J., Book D. // J. Chem. Commun, 2015, pp. 1-3. DOI: 10.1039/C5CC04327A 
14. Naser A. Al-Mufachi, Robert Steinberger-Wilckens // J. Thin Solid Films, 2018, vol. 646, p. 83-91. DOI: 10.1016/j.tsf.2017.11.032

15. Костина А. Е., Волков А. Ю. // Вектор науки, 2017, № 3(41), c. 76-83. DOI: 10.18323/2073-5073-20173-76-83

16. Иевлев В. М., Максименко А. А., Канныкин С. В., Белоногов Е. К., Володин М. С., Рошан Н. Р. // Конденсированные среды и межфазные границы, 2016, т. 18, № 4, c. 521-529.

17. Морозова Н. Б., Введенский А. В., Максименко А. А., Донцов А. И // Конденсированные среды и межфазные гранищь, 2015, т. 17, № 4, с. 459-469.
18. Иевлев В. М., Рошан Н. Р., Белоногов Е. К., Кущев С. Б., Канныкин С. В., Максименко А. А., Донцов А. И., Глазунова Ю. И. // Конденсированные среды и межфразные границы, 2012, т. 14, № 4, с. 422-427.

19. Морозова Н. Б., Введенский А. В., Бередина И. П. // Конденсированные среды и межфазные гранииы, 2014, т. 16, № 2, с. 178-188.

20. Морозова Н. Б., Введенский А. В. // Конденсированные среды и межфазные границы, 2015, т. 17, № 4, c. $451-458$.

21. Антонова М. С., Белоногов Е. К., Донцов А. И., Максименко А. А. // Международный научный журнал Альтернативная энергетика и экология, 2011, № 9, c. $14-16$.

\title{
HYDROGEN PERMEABILITY OF Pd53Cu FILM ALLOYS OF VARIOUS PHASE COMPOSITION
}

\author{
(C) 2018 A. I. Fedoseeva, N. B. Morozova, A. V. Vvedenskii, A. I. Dontsov \\ Voronezh State University, 1 Universitetskaya pl., 394018 Voronezh Russia \\ e-mail:mnb@chem.vsu.ru
}

Received 23.03.2018

\begin{abstract}
The purpose of this work was to reveal the influence of the phase composition and the crystallographic orientation of film samples of $\mathrm{Pd}-\mathrm{Cu}$ solid solution on the parameters of extraction and injection of atomic hydrogen in aqueous solution of sulphuric acid. The study was carried out using a two-step cathode-anode chronoamperometry method. The films of the Pd53Cu alloy of various thickness were obtained by magnetron sputtering. They were characterised by the same phase ( $100 \%$ of the $\beta$-phase of a solid solution) and chemical composition. It was confirmed that during the initial period $(\leq 4 \mathrm{~s}$, the injection of atomic hydrogen proceeds in a mixed diffusion-phase-boundary regime, which later transforms into a purely diffusion mode. The kinetic and diffusion parameters of the stages of phase-boundary penetration of hydrogen and its subsequent solid-phase diffusion were calculated by the cathodic and anodic current decays. It was established that regardless of the thickness of the sample, an increase in the $\beta$ phase fraction in the alloy leads to a marked increase in the diffusion coefficient of atomic hydrogen and, consequently, to an increase in the hydrogen permeability constant. The crystallographic orientation only affects the stage of phase-boundary penetration of hydrogen into the film alloy. The data obtained confirm the preferential movement of hydrogen along the grain body rather than over the interphase boundaries.
\end{abstract}

Keywords: cathode-anode chronoamperometry, $\mathrm{Pd} 53 \mathrm{Cu}, \beta$ phase of $\mathrm{Cu}-\mathrm{Pd}$ solid solution, film electrodes, hydrogen permeability, phase composition, crystallographic orientation of the surface.

DOI: https://doi.org/10.17308/kcmf.2018.20/519

\section{REFERENCES}

1. Morgan Allemand, Manuel. H. Martin, David Reyter, Lionel Roué, Daniel Guay, Carmen Andrei, Gianluigi A. Botton J. Electrochimica Acta, 2011, vol. 56, pp. 7397-7403. DOI: 10.1016/j.electacta.2011.05.052

2. Sloveckij D. I., Chistov E. M., Roshan N. R. Mezhdunarodnyj nauchnyj zhurnal Al ternativnaja jenergetika $i$ jekologija [International Scientific Journal for Alternative Energy and Ecology (ISJAEE)], 2004, no. 1, pp. 43-46.
3. Fuwu Yan, Lei Xu, Yu Wang J. Renewable and Sustainable Energy Reviews, 2018, vol. 82, №1, pp. 1457-1488. DOI: 10.1016/j.rser.2017.05.227

4. Alessandro Cappelletti, Francesco Martelli J. Hydrogen Energy, 2017, vol. 42, pp. 10513-10523. DOI: 10.1016/j.ijhydene.2017.02.104

5. Zhang Ke, Gade Sabina K., Douglas Way J. J. Membrane Science, 2012, vol. 403-404, pp. 78-83. DOI: 10.1016/j.memsci.2012.02.025 
6. Rahimpour M. R., Samimi F., Babapoor A., Tohidian T., Mohebi S. J. Chemical Engineering and Processing, 2017, vol. 121, p. 24-49. DOI: 10.1016/j.cep.2017.07.021

7. Morreale B. D., Ciocco M. V., Howard B. H., Killmeyer R. P., Cugini A. V., Enick R. M. J. Membrane Science, 2004, vol. 241, p. 219-224. DOI: 10.1016/j.memsci.2004.04.033

8. Ramesh Sharma, Yamini Sharma J. Hydrogen Energy, 2015, vol. 40, pp. 14885-14899. DOI: 10.1016/j. ijhydene.2015.09.016

9. Morozov M. M., Potekaev A. I., Klopotov A. A., Markova T. N., Klopotov V. D. Izvestiua vuzov. Chernaya Metallurgiya [Izvestiya. Ferrous Metallurgy], 2015, vol. 58, no. 8. pp. 589-596. DOI: 10.17073/0368-0797-2015-8-589596 (in Russ.)

10. Avdjuhina V. M., Revkevich G. P., Kacnel'son A. A. Poverhnost'. Rentgenovskie, sinhrotronnye $i$ nejtronnye issledovanija [Journal of Surface Investigation: X-ray, Synchrotron and Neutron Techniques], 2006, № 12, pp. 1522. (in Russ.)

11. Volkov A. Ju., Kazancev V. A., Kourov N. I., Kruglikov N. A. Phys. Metals Metallogr., 2008, vol. 106, no. 4, pp. 341-352. DOI: https://doi.org/10.1134/ S0031918X08100037

12. Volkov A. Ju., Kruglikov N. A. Phys. Metals Metallogr., 2008, vol. 105, no. 2, pp. 202-210. DOI: https://doi. org/10.1007/s11508-008-2013-5

13. Nayebossadri S., Speight J., Book D. J. Chem. Commun., 2015, pp. 1-3. DOI: 10.1039/C5CC04327A

14. Naser A. Al-Mufachi, Robert Steinberger-Wilckens J. Thin Solid Films, 2018, vol. 646, pp. 83-91. DOI: 10.1016/ j.tsf.2017.11.032
15. Kostina A. E., Volkov A. Ju. Vektor Nauki, 2017, no. 3(41), pp. 76-83. DOI: 10.18323/2073-5073-2017-3$76-83$

16. Ievlev V. M., Maksimenko A. A., Kannykin S. V., Belonogov E. K., Volodin M. S., Roshan N. R. Condensed Matter and Interphases, 2016, vol. 18, no. 4, pp. 521-529. Available at: http://www.kcmf.vsu.ru/resources/t_18_4_ 2016_009.pdf (in Russ.)

17. Morozova N. B., Vvedenskij A. V., Maksimenko A. A., Dontsov A. I. Condensed Matter and Interphases, 2015, vol. 17, no. 4, pp. 459-469. Available at: http://www.kcmf. vsu.ru/resources/t_17_4_2015_006.pdf (in Russ.)

18. Ievlev V. M., Roshan N. R., Belonogov E. K., Kushhev S. B., Kannykin S. V., Maksimenko A. A., Dontsov A. I., Glazunova Ju. I. Condensed Matter and Interphases, 2012, vol. 14, no. 4, pp. 422-427. Available at: http://www.kcmf. vsu.ru/resources/t_14_4_2012_004.pdf (in Russ.)

19. Morozova N. B., Vvedenskij A. V., Beredina I. P. Condensed Matter and Interphases, 2014, vol. 16, no. 2, pp. 178-188. Available at: http://www.kcmf.vsu.ru/resources/t_16_2_2014_009.pdf (in Russ.)

20. Morozova N. B., Vvedenskij A. V. Condensed Matter and Interphases, 2015, vol. 17, no. 4, pp. 451-458. Available at: http://www.kcmf.vsu.ru/resources/t_17_4_ 2015_005.pdf (in Russ.)

21. Antonova M. S., Belonogov E. K., Dontsov A. I., Maksimenko A. A. Mezhdunarodnyj nauchnyj zhurnal Al'ternativnaja jenergetika i jekologija [International Scientific Journal for Alternative Energy and Ecology (IS$J A E E)$ ], 2011, no. 9, pp. 14-16. (in Russ.)
Федосеева Анастасия Игоревна - студентка кафедры физической химии, химический факультет, Воронежский государственный университет; тел. +7(473) 2208538, e-mail: Kanamepsp@yandex.ru

Морозова Наталья Борисовна - к. х. н., доцент кафедры физической химии, Воронежский государственный университет; тел.+7 (473) 2208538, e-mail: mnb@chem.vsu.ru

Введенский Александр Викторович - д. х. н., профессор, заведующий кафедрой физической химии, Воронежский государственный университет; тел.: +7(473) 2208546, e-mail: alvved@chem.vsu.ru

Донияов Алексей Игоревич - к. ф.-м. н., н. с. кафедры материаловедения и индустрии наносистем, Воронежский государственный университет; тел.: +7(473) 2208735, e-mail: dontalex@mail.ru
Anastasija I. Fedoseeva - student of Department of Physical Chemistry, Chemical Faculty, Voronezh State University; tel.: +7(473) 2208538, e-mail: Kanamepsp@yandex.ru

Natalia B. Morozova - Cand. Sci. (Chem.), Associate Professor, Department of Physical Chemistry, Voronezh State University; tel.: +7(473) 2208538, email:mnb@chem.vsu.ru

Alexander V. Vvedenskii-Dr. Sci. (Chem.), Professor, Head of the Department of Physical Chemistry, Voronezh State University; tel.: +7(473) 2208546, e-mail: alvved@chem.vsu.ru

Aleksey I. Dontsov - Cand. Sci. (Phys.-Math.), Junior Research Associate, Department of Materials Science and Industry Nanosystems, Voronezh State University; tel.: +7(473) 2208735, e-mail: dontalex@mail.ru 